\title{
An Approximation Method for Convolution Calderón-Zygmund Operators
}

\author{
Yunxia $\mathbf{Y u}^{1}$ and Zhanying Yang ${ }^{2,3}$ \\ ${ }^{1}$ Department of Mathematics, Xinxiang University, Xinxiang 453003, China \\ ${ }^{2}$ Department of Mathematics, South-Central University for Nationalities, Wuhan 430074, China \\ ${ }^{3}$ Laboratoire de Mathématiques Raphaaël Salem, UMR 6085 CNRS-Université de Rouen, avenue de l'Université, BP 12, \\ 76801 Saint-Etienne-du-Rouvray, France
}

Correspondence should be addressed to Zhanying Yang; yangzhanying8011@gmail.com

Received 14 December 2012; Accepted 28 May 2013

Academic Editor: Youyu Wang

Copyright (C) 2013 Y. Yu and Z. Yang. This is an open access article distributed under the Creative Commons Attribution License, which permits unrestricted use, distribution, and reproduction in any medium, provided the original work is properly cited.

We present an approximation method for convolution Calderón-Zygmund operators. We give a uniform approximation accuracy of the operators on the endpoint Triebel-Lizorkin space $\dot{F}_{1}^{0 ; q}(2<q<\infty)$. Our proof mainly relies on the $n$-dimensional Daubechies wavelet bases and the atomic-molecular approach.

\section{Introduction and Main Result}

For rapid application of dense matrices (or integral operators) to vectors, the celebrated work of Beylkin et al. [1] introduced a class of numerical algorithms which are based on the $2 n$ dimensional wavelet bases with compact supports. These algorithms are also applicable to all Calderón-Zygmund operators and pseudodifferential operators. Since then, their algorithms are widely used in compression of matrices, operator approximation, and establishing boundedness of operators see [2-9]; In particular, Beylkin et al. [1] approximated a class of Calderón-Zygmund operators by banded operators and gave the approximation accuracy. It is intriguing to know whether we can get some similar approximation methods on some more general spaces. Notice that Yang [9] approximated the operators by compact operators and gave the approximation accuracy on $L^{p}(1<p<\infty)$.

In this paper, we are interested in considering the approximation method for a class of Calderón-Zygmund operators on Triebel-Lizorkin spaces. However, due to technical reasons, we can only get an approximation method for convolution Calderón-Zygmund operators on some endpoint Triebel-Lizorkin spaces (see Theorem 1).

Now, we introduce a class of Calderón-Zygmund operators. Let $\mathscr{D}=\mathscr{D}\left(\mathbb{R}^{n}\right)$ denote the space of Schwartz test functions and $\mathscr{D}^{\prime}$ the space of Schwartz distributions (the dual of $\mathscr{D})$. Suppose that we have a linear continuous mapping $T: \mathscr{D} \rightarrow \mathscr{D}^{\prime}$ associated with a kernel $K(x, y)$ (in the sense that $\langle T f, g\rangle=\iint g(x) K(x, y) f(y) d x d y$ for test functions $f$ and $g$ with disjoint supports). We write $T \in$ $\mathrm{CZO}_{\gamma}$ if the following three conditions are satisfied.

(I) $K(x, y)$ is continuous on $\Omega=\mathbb{R}^{n} \times \mathbb{R}^{n} \backslash\{(x, y): x=y\}$ and satisfies

$$
|K(x, y)| \leq C|x-y|^{-n}
$$

for all $x, x^{\prime}, y$ with $2\left|x-x^{\prime}\right| \leq|x-y|$,

$$
\left|K(x, y)-K\left(x^{\prime}, y\right)\right|+\left|K(y, x)-K\left(y, x^{\prime}\right)\right| \leq \frac{C\left|x-x^{\prime}\right|^{\gamma}}{|x-y|^{n+\gamma}},
$$

where $0<\gamma \leq 1$.

(II) Weak boundedness condition:

$$
\begin{array}{r}
|\langle T f, g\rangle| \leq C R^{n}\left(\|f\|_{\infty}+R\|\nabla f\|_{\infty}\right)\left(\|g\|_{\infty}+R\|\nabla g\|_{\infty}\right), \\
\forall R>0, u \in \mathbb{R}^{n}, f, g \in C_{0}^{1}(B(u, R)) .
\end{array}
$$


(III) $T 1$ condition: $T 1 \in B M O, T^{*} 1 \in B M O$.

Convolution Calderón-Zygmund operators, such as Hilbert and Riesz operators, are commonly used in engineering. For a convolution operator $T$, its kernel $K(x, y)$ can be written as $K(x, y)=K(x-y)$. In this case, the conditions for the operator $T \in \mathrm{CCZO}_{\gamma}$ are reduced to the following:

$$
|K(x)| \leq C|x|^{-n}
$$

for $2\left|x-x^{\prime}\right| \leq|x|$,

$$
\begin{gathered}
\left|K(x)-K\left(x^{\prime}\right)\right| \leq \frac{C\left|x-x^{\prime}\right|^{\gamma}}{|x|^{n+\gamma}} ; \\
\left|\int K(x) \psi_{t}(x) d x\right| \leq C\left(\|\psi\|_{\infty}+\|\nabla \psi\|_{\infty}\right),
\end{gathered}
$$

where $\psi \in C_{0}^{1}(B(0,1))$ and $\psi_{t}(x)=\psi(x / t)$ for $t>0$. For convenience, let $\mathrm{CCZO}_{\gamma}$ denote the collection of all convolution operators in $\mathrm{CZO}_{\gamma}$.

In what follows, we restrict our attention to the operator $T$ in $C_{C Z O}$. In general, the operator is analyzed by the $2 n$-dimensional wavelet bases. However, Z. Y. Yang and Q. $X$. Yang [10], making use of the $n$-dimensional Daubechies wavelet bases, approximated the operator $T$ by the banded operator and gave the approximation accuracy $C 2^{-u \gamma}$ on the homogeneous Besov spaces $\dot{B}_{p}^{0, q}(1 \leq p, q \leq \infty)$. In this paper, we focus on an approximation method for the operator $T$ and obtaining the uniform approximation accuracy on the endpoint Triebel-Lizorkin spaces $\dot{F}_{1}^{0, q}, 2<q<\infty$, whose definitions will be given in Section 2.

We first introduce some notations. Let $\Phi^{0}(x)$ and $\Phi^{1}(x)$ be the one-dimensional Daubechies father and mother wavelets, respectively. Assume that they are the real-valued and sufficiently regular functions. For $x \in \mathbb{R}^{n}$ and $\varepsilon=$ $\left(\varepsilon_{1}, \varepsilon_{2}, \ldots, \varepsilon_{n}\right) \in\{0,1\}^{n}$, denote $\Phi^{\varepsilon}(x)=\prod_{i=1}^{n} \Phi^{\varepsilon_{i}}\left(x_{i}\right)$. For any $j \in \mathbb{Z}$ and $k \in \mathbb{Z}^{n}$, let

$$
\Phi_{j, k}^{\varepsilon}(x)=2^{j n / 2} \Phi^{\varepsilon}\left(2^{j} x-k\right)
$$

We also put

$$
\begin{gathered}
E_{n}=\{0,1\}^{n} \backslash\{0\}, \\
\Lambda_{n}=\left\{\lambda=(\varepsilon, j, k), \varepsilon \in E_{n}, j \in \mathbb{Z}, k \in \mathbb{Z}^{n}\right\} .
\end{gathered}
$$

Then, $\left\{\Phi_{j, k}^{\varepsilon}(x)\right\}_{\lambda \in \Lambda_{n}}$ forms orthonormal bases in $L^{2}\left(\mathbb{R}^{n}\right)$, and it can be used to characterize general functions or distributions.

For any $\lambda \in \Lambda_{n}$, let $b_{j, k}^{\varepsilon}=\left\langle K(x), \Phi_{j, k}^{\varepsilon}(x)\right\rangle$, then we have the representation

$$
K=\sum_{(\varepsilon, j, k) \in \Lambda_{n}} b_{j, k}^{\varepsilon} \Phi_{j, k}^{\varepsilon}(x)
$$

in the sense of distribution. Now, we present the approximation of $T$ by the banded operator $T_{u}$. For any integer $u \geq 0$ and $\varepsilon \in E_{n}$, we define

$$
K_{u}^{\varepsilon}(x)= \begin{cases}\sum_{j} b_{j, 0}^{\varepsilon} \Phi_{j, 0}^{\varepsilon}(x), & u=0 ; \\ \sum_{2^{u-1} \leq|k|<2^{u}} \sum_{j} b_{j, k}^{\varepsilon} \Phi_{j, k}^{\varepsilon}(x), & u \geq 1 .\end{cases}
$$

Let $T_{u}^{\varepsilon}$ be the annular operator associated to the $\operatorname{kernel} K_{u}^{\varepsilon}(x)$, and let

$$
T_{u}=\sum_{0 \leq u^{\prime} \leq u} \sum_{\varepsilon \in E_{n}} T_{u^{\prime}}^{\varepsilon}
$$

Then, we can approximate $T$ by the banded operator $T_{u}$ and get the uniform approximation accuracy on the endpoint Triebel-Lizorkin space $\dot{F}_{1}^{0, q}(1<q<\infty)$. Our result is stated as follows.

Theorem 1. Let $1<q<\infty$ and $0<\gamma \leq 1$. If $T \in C C Z O_{\gamma}$, then

$$
\left\|T-T_{u}\right\|_{\dot{F}_{1}^{0, q} \rightarrow \dot{F}_{1}^{0, q}} \leq \mathrm{Cu} 2^{-u \gamma}, \quad u \geq 1 .
$$

Throughout this paper, the symbol $C$ denotes a constant that is independent of the main parameters involved but whose value may differ from line to line.

\section{Endpoint Triebel-Lizorkin Spaces}

Let $\delta\left(\mathbb{R}^{n}\right)$ be the space of tempered test functions. Let $\varphi \in$ $\mathcal{S}\left(\mathbb{R}^{n}\right)$ with supp $\widehat{\varphi} \subset\left\{\xi \in \mathbb{R}^{n}: 1 / 2 \leq|\xi| \leq 2\right\}$ and $|\widehat{\varphi}| \geq c>0$ for $\{3 / 5 \leq|\xi| \leq 5 / 3\}$; let $\varphi_{j}(x) \equiv 2^{j n} \varphi\left(2^{j} x\right)$. Let $\alpha \in \mathbb{R}$, $0<p<\infty$, and $0<q \leq \infty$. Then, the homogeneous TriebelLizorkin space $\dot{F}_{p}^{\alpha, q}$ is defined as the collection of all $f \in \mathcal{S}^{\prime} / P$ (the tempered distributions modulo polynomials) such that

$$
\|f\|_{\dot{F}_{p}^{\alpha, q}} \equiv\left\|\left(\sum_{j}\left(2^{j \alpha}\left|\varphi_{j} * f\right|\right)^{q}\right)^{1 / q}\right\|_{p}<\infty
$$

with the usual modification made when $q=\infty$; see also Triebel [11]. And it is well known that the homogeneous Triebel-Lizorkin space and Besov space are the same when $p=q$.

For the homogeneous Triebel-Lizorkin space, Koskela et al. gave the characterization via grand Littlewood-Paley functions in [12] and gave the pointwise characterization in [13]. Now, we recall its characterization based on wavelets. In fact, the Daubechies wavelet bases $\left\{\Phi_{j, k}^{\varepsilon}(x)\right\}_{\lambda \in \Lambda_{n}}$ is an unconditional bases in the space $\dot{F}_{p}^{0, q}$. For any $j \in \mathbb{Z}$ and $k \in \mathbb{Z}^{n}$, let

$$
Q_{j, k}=\left\{x \in \mathbb{R}^{n}: 2^{j} x-k \in[0,1)^{n}\right\},
$$

and let $\chi\left(2^{j} x-k\right)$ be the characteristic function of the dyadic cube $Q_{j, k}$. For any $f \in \mathcal{S}^{\prime} / P$, if we can define $f_{j, k}^{\varepsilon}=\left\langle f, \Phi_{j, k}^{\varepsilon}\right\rangle$ for all $(\varepsilon, j, k) \in \Lambda_{n}$, then we have the representation $f=$ $\sum_{(\varepsilon, j, k) \in \Lambda_{n}} f_{j, k}^{\varepsilon} \Phi_{j, k}^{\varepsilon}(x)$ in the sense of distribution. The space $\dot{F}_{p}^{0, q}$ is characterized in terms of wavelets in the following way (for more details, see also $[6,7,14]$ ). 
Proposition 2. For $1 \leq p<\infty$ and $1 \leq q \leq \infty$, there exist two positive constants $C_{p, q}$ and $C_{p, q}^{\prime}$ such that

$$
\begin{aligned}
C_{p, q}\|f\|_{\dot{F}_{p}^{0, q}} & \leq\left\|\left(\sum_{\varepsilon, j, k} 2^{j q n / 2}\left|f_{j, k}^{\varepsilon}\right|^{q} \chi\left(2^{j} x-k\right)\right)^{1 / q}\right\|_{L^{p}} \\
& \leq C_{p, q}^{\prime}\|f\|_{\dot{F}_{p}^{0, q} .}
\end{aligned}
$$

Triebel-Lizorkin spaces have been studied by means of the atomic and molecular decompositions. Next, we state the atomic-molecular decomposition for the endpoint space $\dot{F}_{1}^{0, q}$; see Meyer and Yang [7] for more details. Let $\mathfrak{Q}$ denote the collection of all dyadic cubes $Q_{j, k}, j \in \mathbb{Z}, k \in \mathbb{Z}^{n}$. Now, we recall the following two definitions which can be found in $[6,7,15]$.

Definition 3. Let $1 \leq q<\infty$. $a$ is said to be a $\dot{F}_{q}^{0, q}$-atom in $\dot{F}_{1}^{0, q}$ with the norm $C$ if there exists a ball $B$ such that

(i) supp $a \subset B$;

(ii) $\|a\|_{\dot{F}_{q}^{0, q}} \leq C|B|^{(1 / q)-1}$;

(iii) $\int a d x=0$.

Definition 4. Let $1 \leq q<\infty . a$ is said to be a $\dot{F}_{q}^{0, q}$-molecule in $\dot{F}_{1}^{0, q}$ with the norm $C$ if there exists a cube $Q_{s, p} \in \mathfrak{Q}$ such that

(i) $a=\sum_{\substack{(\varepsilon, j, k) \in \Lambda_{n} \\ \mathbb{Q}_{j, k} \subset Q_{s, p}}}^{\varepsilon} a_{j, k}^{\varepsilon} \Phi_{j, k}^{\varepsilon}$;

(ii) $\|a\|_{\dot{F}_{q}^{0, q}} \leq C\left|Q_{s, p}\right|^{(1 / q)-1}$.

For convenience, in the above two definitions, $a$ is said to be a $\dot{F}_{q}^{0, q}$-atom or a $\dot{F}_{q}^{0, q}$-molecule when $C=1$.

Proposition 5. Let $1 \leq q<\infty$. The following three conditions are equivalent:

(i) $f \in \dot{F}_{1}^{0, q}$.

(ii) There exist $\left\{\mu_{m}\right\}_{m \in \mathbb{Z}} \in l^{1}$ and $\dot{F}_{q}^{0, q}$-atoms $a_{m}$ such that $f=\sum_{m \in \mathbb{Z}} \mu_{m} a_{m}$.

(iii) There exist $\left\{\lambda_{m}\right\}_{m \in \mathbb{Z}} \in l^{1}$ and $\dot{F}_{q}^{0, q}$-molecules $a_{m}$ such that $f=\sum_{m \in \mathbb{Z}} \lambda_{m} a_{m}$.

In fact, for any $f \in \dot{F}_{1}^{0, q}$, we can write it as $f=\sum_{m} \lambda_{m} a_{m}$, where each $a_{m}$ is a $\dot{F}_{q}^{0, q}$-molecule and $\left\{\lambda_{m}\right\} \in l^{1}$, and it can be verified that

$$
\|f\|_{\dot{F}_{1}^{0, q}} \sim \sum_{m}\left|\lambda_{m}\right|
$$

namely,

$$
C_{1}\|f\|_{\dot{F}_{1}^{0, q}} \leq \sum_{m}\left|\lambda_{m}\right| \leq C_{2}\|f\|_{\dot{F}_{1}^{0, q}}
$$

for some fixed $C_{1}, C_{2}>0$ independent of $f$. For more details, we refer the reader to $[6,15]$, This will play a key role in Section 3 .

\section{Estimate of $T_{u}^{\varepsilon^{\prime}}$ and Proof of Theorem 1}

To prove Theorem 1, we first estimate the annular operator $T_{u}^{\varepsilon^{\prime}}$. For any $\varepsilon^{\prime} \in E_{n}$ and integer $u \geq 1$, let

$$
B_{u}^{\varepsilon^{\prime}}=\sup _{j^{\prime}} \sum_{2^{u-1} \leq\left|k^{\prime}\right|<2^{u}} 2^{-j^{\prime} n / 2}\left|b_{j^{\prime}, k^{\prime}}^{\varepsilon^{\prime}}\right| .
$$

Lemma 6. For $1<q<\infty$, let $\tau_{q}=1-(1 / q)$. One has

$$
\left\|T_{u}^{\varepsilon^{\prime}}\right\|_{\dot{F}_{1}^{0, q} \rightarrow \dot{F}_{1}^{0, q}} \leq C u^{\tau_{q}} B_{u}^{\varepsilon^{\prime}} .
$$

Proof. In terms of the molecular decomposition for the space $\dot{F}_{1}^{0, q}$, we only need to prove that for an arbitrary $\dot{F}_{q}^{0, q}$-molecule $a$,

$$
\left\|T_{u}^{\varepsilon^{\varepsilon^{\prime}}} a(x)\right\|_{\dot{F}_{1}^{0, q}} \leq C u^{\tau_{q}} B_{u}^{\varepsilon^{\prime}},
$$

where $C$ is independent of $a$.

Denote $m=j^{\prime}-j$, then we have

$$
\begin{aligned}
T_{u}^{\varepsilon^{\prime}} a(x)=\sum_{\varepsilon, m} \sum_{2^{u-1} \leq\left|k^{\prime}\right|<2^{u}} & \\
& \times \sum_{Q_{j, k}<Q_{s, p}} b_{j+m, k^{\prime}}^{\varepsilon^{\prime}} a_{j, k}^{\varepsilon}\left(\Phi_{j, k}^{\varepsilon} * \Phi_{j+m, k^{\prime}}^{\varepsilon^{\prime}}\right)(x) .
\end{aligned}
$$

Thus,

$$
\begin{aligned}
& \left\|T_{u}^{\varepsilon^{\prime}} a(x)\right\|_{\dot{F}_{1}^{0, q}} \\
& \leq \sum_{\varepsilon} \| \sum_{m \leq 0} \sum_{2^{u-1} \leq\left|k^{\prime}\right|<2^{u}} \\
& \quad \times \sum_{Q_{j, k} \subset Q_{s, p}} b_{j+m, k^{\prime}}^{\varepsilon^{\prime}} a_{j, k}^{\varepsilon}\left(\Phi_{j, k}^{\varepsilon} * \Phi_{j+m, k^{\prime}}^{\varepsilon^{\prime}}\right)(x) \|_{\dot{F}_{1}^{0, q}} \\
& +\sum_{\varepsilon} \| \sum_{m>0} \sum_{2^{u-1} \leq\left|k^{\prime}\right|<2^{u}} \\
& \quad \times \sum_{Q_{j, k} \subset Q_{s, p}} b_{j+m, k^{\prime}}^{\varepsilon^{\prime}} a_{j, k}^{\varepsilon}\left(\Phi_{j, k}^{\varepsilon} * \Phi_{j+m, k^{\prime}}^{\varepsilon^{\prime}}\right)(x) \|_{\dot{F}_{1}^{0, q}}
\end{aligned}
$$$$
:=I_{1}+I_{2} \text {. }
$$

In the following, we consider the estimates of $I_{1}$ and $I_{2}$.

(i) For any $m \leq 0$, put $\widetilde{\Phi}_{m}^{\varepsilon, \varepsilon^{\prime}}(x)=2^{-m n}\left(\Phi_{m, 0}^{\varepsilon^{\prime}} * \Phi^{\varepsilon}\right)(x)$, then

$$
\begin{aligned}
I_{1} \leq & C \sum_{m \leq 0} 2^{m n} \sum_{2^{u-1} \leq\left|k^{\prime}\right|<2^{u}} \\
& \times\left\|\sum_{Q_{j, k} \subset Q_{s, p}} b_{j+m, k^{\prime}}^{\varepsilon^{\prime}} a_{j, k}^{\varepsilon} \widetilde{\Phi}_{m}^{\varepsilon, \varepsilon^{\prime}}\left(2^{j} x-\left(k+2^{-m} k^{\prime}\right)\right)\right\|_{\dot{F}_{1}^{0, q}} .
\end{aligned}
$$


For fixed $m$ and $k^{\prime}$, let $T_{k^{\prime}}^{m}$ be the operator associated with the kernel

$$
\begin{aligned}
K_{k^{\prime}}^{m}(x, y)= & \sum_{Q_{j, k} \subset Q_{s, p}} 2^{j n} \widetilde{\Phi}_{m}^{\varepsilon, \varepsilon^{\prime}}\left(2^{j} x-\left(k+2^{-m} k^{\prime}\right)\right) \\
& \times \Phi^{\varepsilon}\left(2^{j} y-\left(k+2^{-m} k^{\prime}\right)\right) .
\end{aligned}
$$

By the properties of Daubechies wavelets, we can get that the operator $T_{k^{\prime}}^{m}$ is bounded on $\dot{F}_{1}^{0, q}$ (see also [16, lemma 3.1]).

Set

$$
g_{k^{\prime}}^{m}=\sum_{Q_{j, k} \subset Q_{s, p}} b_{j+m, k^{\prime}}^{\varepsilon^{\prime}} a_{j, k}^{\varepsilon} \Phi^{\varepsilon}\left(2^{j} x-\left(k+2^{-m} k^{\prime}\right)\right) .
$$

By means of the orthonormality of the wavelet bases, the right side of (23) is equal to

$$
C \sum_{m \leq 0} 2^{m n} \sum_{2^{u-1} \leq\left|k^{\prime}\right|<2^{u}}\left\|T_{k^{\prime}}^{m} g_{k^{\prime}}^{m}\right\|_{\dot{F}_{1}^{0, q}}
$$

Moreover, $I_{1}$ is bounded by

$$
C \sum_{m \leq 0} 2^{m n} \sum_{2^{u-1} \leq\left|k^{\prime}\right|<2^{u}}\left\|g_{k^{\prime}}^{m}\right\|_{\dot{F}_{1}^{0, q}}
$$

Namely, we have

$$
\begin{aligned}
I_{1} \leq & C \sum_{m \leq 0} 2^{m n} \sum_{2^{u-1} \leq\left|k^{\prime}\right|<2^{u}} \\
& \times\left\|_{Q_{j, k} \subset Q_{s, p}} b_{j+m, k^{\prime}}^{\varepsilon^{\prime}} a_{j, k}^{\varepsilon} \Phi^{\varepsilon}\left(2^{j} x-\left(k+2^{-m} k^{\prime}\right)\right)\right\|_{\dot{F}_{1}^{0, q}} .
\end{aligned}
$$

Notice that the sum $\sum_{j: Q_{j, k} \subset Q_{s, p}}$ is adding among all $j$ with $s \leq j$, we split $\sum_{j: Q_{j, k} \subset Q_{s, p}}$ into $\sum_{j: Q_{j, k} \subset Q_{s, p}}=\sum_{s \leq j<s+u}+\sum_{j \geq s+u}$. Hence, we obtain

$$
\begin{aligned}
I_{1} \leq C \sum_{m \leq 0} 2^{m n} & \\
\times \sum_{2^{u-1} \leq\left|k^{\prime}\right|<2^{u}} & \left(\| \sum_{\substack{s \leq j<s+u \\
Q_{j, k} \subset Q_{s, p}}} b_{j+m, k^{\prime}}^{\varepsilon^{\prime}} a_{j, k}^{\varepsilon} \Phi^{\varepsilon}\right. \\
& \times\left(2^{j} x-\left(k+2^{-m} k^{\prime}\right)\right)
\end{aligned}
$$

$$
+\sum_{\substack{j \geq s+u \\ Q_{j, k} \subset Q_{s, p}}} b_{j+m, k^{\prime}}^{\varepsilon^{\prime}} a_{j, k}^{\varepsilon} \Phi^{\varepsilon}
$$$$
\left.\times\left(2^{j} x-\left(k+2^{-m} k^{\prime}\right)\right)\|\|_{\dot{F}_{1}^{0, q}}\right)
$$$$
:=C \sum_{m \leq 0} 2^{m n} \sum_{2^{u-1} \leq\left|k^{\prime}\right|<2^{u}}\left(J_{1, k^{\prime}}^{m, u}+J_{2, k^{\prime}}^{m, u}\right)
$$

Now, we consider $J_{1, k^{\prime}}^{m, u}$ and $J_{2, k^{\prime}}^{m, u}$. As for $J_{1, k^{\prime}}^{m, u}$, we use Proposition 2 to get that

$$
\begin{aligned}
& J_{1, k^{\prime}}^{m, u} \leq C \int\left(\sum_{\substack{s \leq j<s+u \\
Q_{j, k} \subset Q_{s, p}}}\left|b_{j+m, k^{\prime}}^{\varepsilon^{\prime}}\right|^{q}\right. \\
& \left.\quad \times\left|a_{j, k}^{\varepsilon}\right|^{q} \chi\left(2^{j} x-\left(k+2^{-m} k^{\prime}\right)\right)\right)^{1 / q} d x \\
& \leq C 2^{m n / 2} \sup _{j^{\prime}} 2^{-j^{\prime} n / 2}\left|b_{j^{\prime}, k^{\prime}}^{\varepsilon^{\prime}}\right|
\end{aligned}
$$

$$
\times \int\left(\sum_{\substack{s \leq j<s+u \\ Q_{j, k} \subset Q_{s, p}}} 2^{j q n / 2}\left|a_{j, k}^{\varepsilon}\right|^{q}\right.
$$

$$
\left.\times \chi\left(2^{j} x-\left(k+2^{-m} k^{\prime}\right)\right)\right)^{1 / q} d x
$$

Let $\otimes_{1}^{n}\left[a_{i}, b_{i}\right]$ denote the Cartesian product $\left[a_{1}, b_{1}\right] \times\left[a_{2}, b_{2}\right] \times$ $\cdots \times\left[a_{n}, b_{n}\right]$. Let

$$
f_{j, k^{\prime}}^{m}(x)=\sum_{k: Q_{j, k} \subset Q_{s, p}} 2^{j q n / 2}\left|a_{j, k}^{\varepsilon}\right|^{q} \chi\left(2^{j} x-\left(k+2^{-m} k^{\prime}\right)\right),
$$

then the support of $f_{j, k^{\prime}}^{m}(x)$ is contained in $\otimes_{1}^{n}\left[\left(p_{i} / 2^{s}\right)+\right.$ $\left.\left(k_{i}^{\prime} / 2^{j+m}\right),\left(\left(p_{i}+1\right) / 2^{s}\right)+\left(k_{i}^{\prime} / 2^{j+m}\right)\right]$. Moreover, we can obtain that

$$
\operatorname{supp} f_{j, k^{\prime}}^{m}(x) \cap \operatorname{supp} f_{j_{1}, k^{\prime}}^{m}(x)=\emptyset
$$

when $\left|j-j_{1}\right| \geq C$. In other words, the number of the nonzero terms in the sum $\sum_{s \leq j<s+u} f_{j, k^{\prime}}^{m}(x)$ is a constant, which is 
independent of $u$. By the inequality $(\alpha+\beta)^{1 / q} \leq \alpha^{1 / q}+\beta^{1 / q}$, we have

$$
\begin{aligned}
J_{1, k^{\prime}}^{m, u} \leq & C 2^{m n / 2} \sup _{j^{\prime}} 2^{-j^{\prime} n / 2}\left|b_{j^{\prime}, k^{\prime}}^{\varepsilon^{\prime}}\right| \\
& \times \int_{\substack{s \leq j<s+u \\
Q_{j, k} \subset Q_{s, p}}} 2^{j n / 2}\left|a_{j, k}^{\varepsilon}\right| \chi\left(2^{j} x-\left(k+2^{-m} k^{\prime}\right)\right) d x \\
\leq & C 2^{m n / 2} \sup _{j^{\prime}} 2^{-j^{\prime} n / 2}\left|b_{j^{\prime}, k^{\prime}}^{\varepsilon^{\prime}}\right| \\
& \times \int_{\substack{s \leq j<s+u \\
Q_{j, k} \subset Q_{s, p}}} 2^{j n / 2}\left|a_{j, k}^{\varepsilon}\right| \chi\left(2^{j} x-k\right) d x .
\end{aligned}
$$

From Hölder's inequality, we obtain

$$
\begin{aligned}
J_{1, k^{\prime}}^{m, u} \leq & C 2^{m n / 2} \sup _{j^{\prime}} 2^{-j^{\prime} n / 2}\left|b_{j^{\prime}, k^{\prime}}^{\varepsilon^{\prime}}\right| u^{\tau_{q}} \\
& \times \int\left(\sum_{s \leq j<s+u}\right. \\
& \left.\times\left(\sum_{k: Q_{j, k} \subset Q_{s, p}} 2^{j n / 2}\left|a_{j, k}^{\varepsilon}\right| \chi\left(2^{j} x-k\right)\right)^{q}\right)^{1 / q} d x \\
\leq & C 2^{m n / 2} \sup _{j^{\prime}} 2^{-j^{\prime} n / 2}\left|b_{j^{\prime}, k^{\prime}}^{\varepsilon^{\prime}}\right| u^{\tau_{q}} \\
& \times \int\left(\sum_{s \leq j<s+u} 2_{Q_{j, k}^{j} \subset Q_{s, p}}^{1 / q n / 2}\left|a_{j, k}^{\varepsilon}\right|^{q} \chi\left(2^{j} x-k\right)\right)^{1 / q} d x .
\end{aligned}
$$

Since $\|a\|_{\dot{F}_{q}^{0, q q}} \leq C|B|^{1 / q-1}$, then we get that $\|a\|_{\dot{F}_{1}^{0, q}} \leq C$. Thus

$$
J_{1, k^{\prime}}^{m, u} \leq C 2^{m n / 2} u^{\tau_{q}} \sup _{j^{\prime}} 2^{-j^{\prime} n / 2}\left|b_{j^{\prime}, k^{\prime}}^{\varepsilon^{\prime}}\right| .
$$

As for $J_{2, k^{\prime}}^{m, u}$, we have

$$
\begin{aligned}
J_{2, k^{\prime}}^{m, u} \leq & C 2^{m n / 2} \sup _{j^{\prime}} 2^{-j^{\prime} n / 2}\left|b_{j^{\prime}, k^{\prime}}^{\varepsilon^{\prime}}\right| \\
& \times\left\|\sum_{\substack{j \geq s+u \\
Q_{j, k} c Q_{s, p}}} 2^{j n / 2} a_{j, k}^{\varepsilon} \Phi^{\varepsilon}\left(2^{j} x-\left(k+2^{-m} k^{\prime}\right)\right)\right\|_{\dot{F}_{1}^{0, q}} \\
:= & C 2^{m n / 2} \sup _{j^{\prime}} 2^{-j^{\prime} n / 2}\left|b_{j^{\prime}, k^{\prime}}^{\varepsilon^{\prime}}\right|\left\|h_{u, k^{\prime}}^{m}(x)\right\|_{\dot{F}_{1}^{0, q} .} .
\end{aligned}
$$

By Definition 3, we can verify that $\left(2^{-m}+2^{M}\right)^{-\tau_{q}{ }^{n}} h_{u, k^{\prime}}^{m}(x)$ is a $\dot{F}_{q}^{0, q}$-atom with the norm $C$, see also [16] for more details. Furthermore, we obtain $\left\|h_{u, k^{\prime}}^{m}(x)\right\|_{\dot{F}_{1}^{0, q}} \leq\left(2^{-m}+2^{M}\right)^{\tau_{q}{ }^{n}}$. Hence,

$$
J_{2, k^{\prime}}^{m, u} \leq C 2^{m n / 2} \sup _{j^{\prime}} 2^{-j^{\prime} n / 2}\left|b_{j^{\prime}, k^{\prime}}^{\varepsilon^{\prime}}\right|\left(2^{-m}+2^{M}\right)^{\tau_{q} n} .
$$

By (29) and (35), it follows that

$$
I_{1} \leq C \sum_{m \leq 0} 2^{3 m n / 2} u^{\tau_{q}} B_{u}^{\varepsilon^{\prime}} \leq C u^{\tau_{q}} B_{u}^{\varepsilon^{\prime}} .
$$

(ii) The estimate for $I_{2}$ can be treated as that for $I_{1}$. For convenience of the reader, we repeat some details as follows. Let $\widetilde{\Phi}_{m}^{\varepsilon, \varepsilon^{\prime}}(x)=2^{m n}\left(\Phi_{m, 0}^{\varepsilon^{\prime}} * \Phi^{\varepsilon}\right)\left(2^{-m} x\right)$. Following the idea used to get (28), we have

$$
\begin{aligned}
I_{2} \leq & C \sum_{m>0} 2^{-m n} \sum_{2^{u-1} \leq\left|k^{\prime}\right|<2^{u}} \\
& \times\left\|\sum_{Q_{j, k} \subset Q_{s, p}} b_{j+m, k^{\prime}}^{\varepsilon^{\prime}} a_{j, k}^{\varepsilon} \Phi^{\varepsilon}\left(2^{j+m} x-\left(k^{\prime}+2^{m} k\right)\right)\right\|_{\dot{F}_{1}^{0, q}} .
\end{aligned}
$$

Splitting $\sum_{j: Q_{j, k} \subset Q_{s, p}}$ into $\sum_{\substack{s \leq j<s+u-m \\ Q_{j, k} \subset Q_{s, p}}}$ and $\sum_{\substack{j \geq s+u-m \\ Q_{j, k} \subset Q_{s, p}}}$ then

$$
\begin{aligned}
& I_{2} \leq C \sum_{m>0} 2^{-m n} \sum_{2^{u-1} \leq\left|k^{\prime}\right|<2^{u}} \\
& \times\left(\left\|\sum_{\substack{s \leq j<s+u-m \\
Q_{j, k} \subset Q_{s, p}}} b_{j+m, k^{\prime}}^{\varepsilon^{\prime}} a_{j, k}^{\varepsilon} \Phi^{\varepsilon}\left(2^{j} x-\left(k+2^{-m} k^{\prime}\right)\right)\right\|_{\dot{F}_{1}^{0, q}}\right. \\
& +\sum_{\substack{j \geq s+u-m \\
Q_{j, k} \subset Q_{, p}}} b_{j+m, k^{\prime}}^{\varepsilon^{\prime}} a_{j, k}^{\varepsilon} \Phi^{\varepsilon} \\
& \left.\times\left(2^{j} x-\left(k+2^{-m} k^{\prime}\right)\right) \|_{\dot{F}_{1}^{0, q}}\right) \\
& :=C \sum_{m>0} 2^{-m n} \sum_{2^{u-1} \leq\left|k^{\prime}\right|<2^{u}}\left(L_{1, k^{\prime}}^{m, u}+L_{2, k^{\prime}}^{m, u}\right) \text {. }
\end{aligned}
$$

The estimates of $L_{1, k^{\prime}}^{m, u}$ and $L_{2, k^{\prime}}^{m, u}$ can be obtained as we handle $J_{1, k^{\prime}}^{m, u}$ and $J_{2, k^{\prime}}^{m, u}$, respectively. In conclusion, it follows that

$$
I_{2} \leq C u^{\tau_{q}} B_{u}^{\varepsilon^{\prime}} .
$$

This completes the proof of Lemma 6 .

In addition to the estimate of the annular operator $T_{u}^{\varepsilon^{\prime}}$, we need the estimate for wavelet coefficients of $K(x)$, which can be found in [10]. 
Proposition 7. Let $K(x)$ be the distribution kernel associated to $T \in C C Z O_{\gamma}$, then one has

$$
\left|b_{j^{\prime}, k^{\prime}}^{\varepsilon^{\prime}}\right| \leq \frac{C 2^{j^{\prime} n / 2}}{\left(1+\left|k^{\prime}\right|\right)^{n+\gamma}}, \quad\left(\varepsilon^{\prime}, j^{\prime}, k^{\prime}\right) \in \Lambda_{n} .
$$

We are now in a position to prove Theorem 1. By virtue of (42), we obtain that

$$
B_{u}^{\varepsilon^{\prime}}=\sup _{j^{\prime}} \sum_{2^{u-1} \leq\left|k^{\prime}\right|<2^{u}} 2^{-j^{\prime} n / 2}\left|b_{j^{\prime}, k^{\prime}}^{\varepsilon^{\prime}}\right| \leq C 2^{-u \gamma} .
$$

Together with Lemma 6, we have

$$
\begin{aligned}
\left\|T-T_{u}\right\|_{\dot{F}_{1}^{0, q} \rightarrow \dot{F}_{1}^{0, q}} & \leq \sum_{u^{\prime}>u} \sum_{\varepsilon^{\prime}}\left\|T_{u^{\prime}}\right\|_{\dot{F}_{1}^{0, q} \rightarrow \dot{F}_{1}^{0, q}} \\
& \leq C \sum_{u l=u+1}^{+\infty}\left(u^{\prime}\right)^{\tau_{q}} 2^{-u^{\prime} \gamma} .
\end{aligned}
$$

On the other hand, since $\tau_{q}$ belongs to the interval $(0,1)$, then a direct computation leads to that

$$
\left\|T-T_{u}\right\|_{\dot{F}_{1}^{0, q} \rightarrow \dot{F}_{1}^{0, q}} \leq C u 2^{-u \gamma} .
$$

This completes the proof of Theorem 1.

Remark 8. Notice that the atomic and molecular decompositions for the endpoint space $\dot{F}_{1}^{0, q}$ play an important role in our proof. However, up to the best knowledge of the authors, it is unknown whether the Triebel-Lizorkin space $\dot{F}_{p}^{s, q}(s \in \mathbb{R}, 1<$ $p, q<\infty)$ has similar atomic and molecular decompositions. It would be interesting to know whether our method can be adjusted to get the approximation accuracy for the more general Triebel-Lizorkin space $\dot{F}_{p}^{s, q}(s \in \mathbb{R}, 1<p, q<\infty)$.

\section{Acknowledgments}

This research is supported by the Fundamental Research Funds for the Central Universities, South-Central University for Nationalities (no. CZY12015; no. CTZ13026), and the Research Fund for the Doctoral Program of Higher Education (no. 20090141120010).

\section{References}

[1] G. Beylkin, R. Coifman, and V. Rokhlin, "Fast wavelet transforms and numerical algorithms. I," Communications on Pure and Applied Mathematics, vol. 44, no. 2, pp. 141-183, 1991.

[2] P. Auscher and Q. X. Yang, "BCR algorithm and the $T(b)$ theorem," Publicacions Matemàtiques, vol. 53, no. 1, pp. 179-196, 2009.

[3] D. G. Deng, L. X. Yan, and Q. X. Yang, "Blocking analysis and $T(1)$ theorem," Science in China. Series A, vol. 41, no. 8, pp. 801808, 1998.

[4] B. Engquist, S. Osher, and S. Zhong, "Fast wavelet based algorithms for linear evolution equations," SIAM Journal on Scientific Computing, vol. 15, no. 4, pp. 755-775, 1994.
[5] F. Keinert, "Biorthogonal wavelets for fast matrix computations," Applied and Computational Harmonic Analysis, vol. 1, no. 2, pp. 147-156, 1994.

[6] K.-S. Lau and L. Yan, "Wavelet decomposition of CalderónZygmund operators on function spaces," Journal of the Australian Mathematical Society, vol. 77, no. 1, pp. 29-46, 2004.

[7] Y. Meyer and Q. X. Yang, "Continuity of Calderón-Zygmund operators on Besov or Triebel-Lizorkin spaces," Analysis and Applications, vol. 6, no. 1, pp. 51-81, 2008.

[8] M. C. Recchioni and F. Zirilli, "The use of wavelets in the operator expansion method for time-dependent acoustic obstacle scattering," SIAM Journal on Scientific Computing, vol. 25, no. 4, pp. 1158-1186, 2003.

[9] Q. X. Yang, "Singular integral operators and approximation by wavelets," Advances in Mathematics, vol. 32, no. 5, pp. 547-552, 2003.

[10] Z. Y. Yang and Q. X. Yang, "Convolution-type CalderónZygmund operators and their approximation," Acta Mathematica Sinica, vol. 51, no. 6, pp. 1061-1072, 2008.

[11] H. Triebel, Theory of Function Spaces, vol. 78, Birkhäuser Verlag, Basel, Switzerland, 1983.

[12] P. Koskela, D. Yang, and Y. Zhou, "A characterization of HajłaszSobolev and Triebel-Lizorkin spaces via grand LittlewoodPaley functions," Journal of Functional Analysis, vol. 258, no. 8, pp. 2637-2661, 2010.

[13] P. Koskela, D. Yang, and Y. Zhou, "Pointwise characterizations of Besov and Triebel-Lizorkin spaces and quasiconformal mappings," Advances in Mathematics, vol. 226, no. 4, pp. 3579-3621, 2011.

[14] D. D. Haroske and H. Triebel, "Wavelet bases and entropy numbers in weighted function spaces," Mathematische Nachrichten, vol. 278, no. 1-2, pp. 108-132, 2005.

[15] Y.-S. Han, M. Paluszyński, and G. Weiss, "A new atomic decomposition for the Triebel-Lizorkin spaces," in Harmonic Analysis and Operator Theory, vol. 189 of Contemporary Mathematics, pp. 235-249, 1995.

[16] Z. Yang, "Boundedness of convolution-type operators on certain endpoint Triebel-Lizorkin spaces," Acta Applicandae Mathematicae, vol. 114, no. 3, pp. 193-205, 2011. 


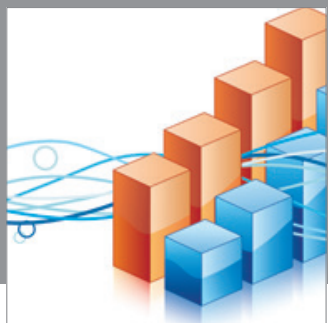

Advances in

Operations Research

mansans

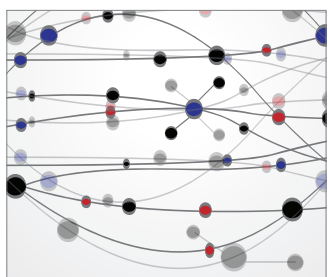

The Scientific World Journal
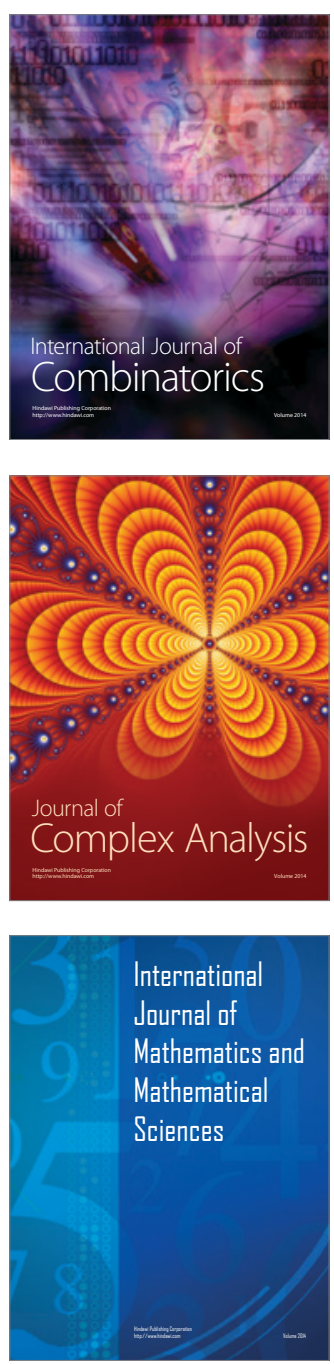
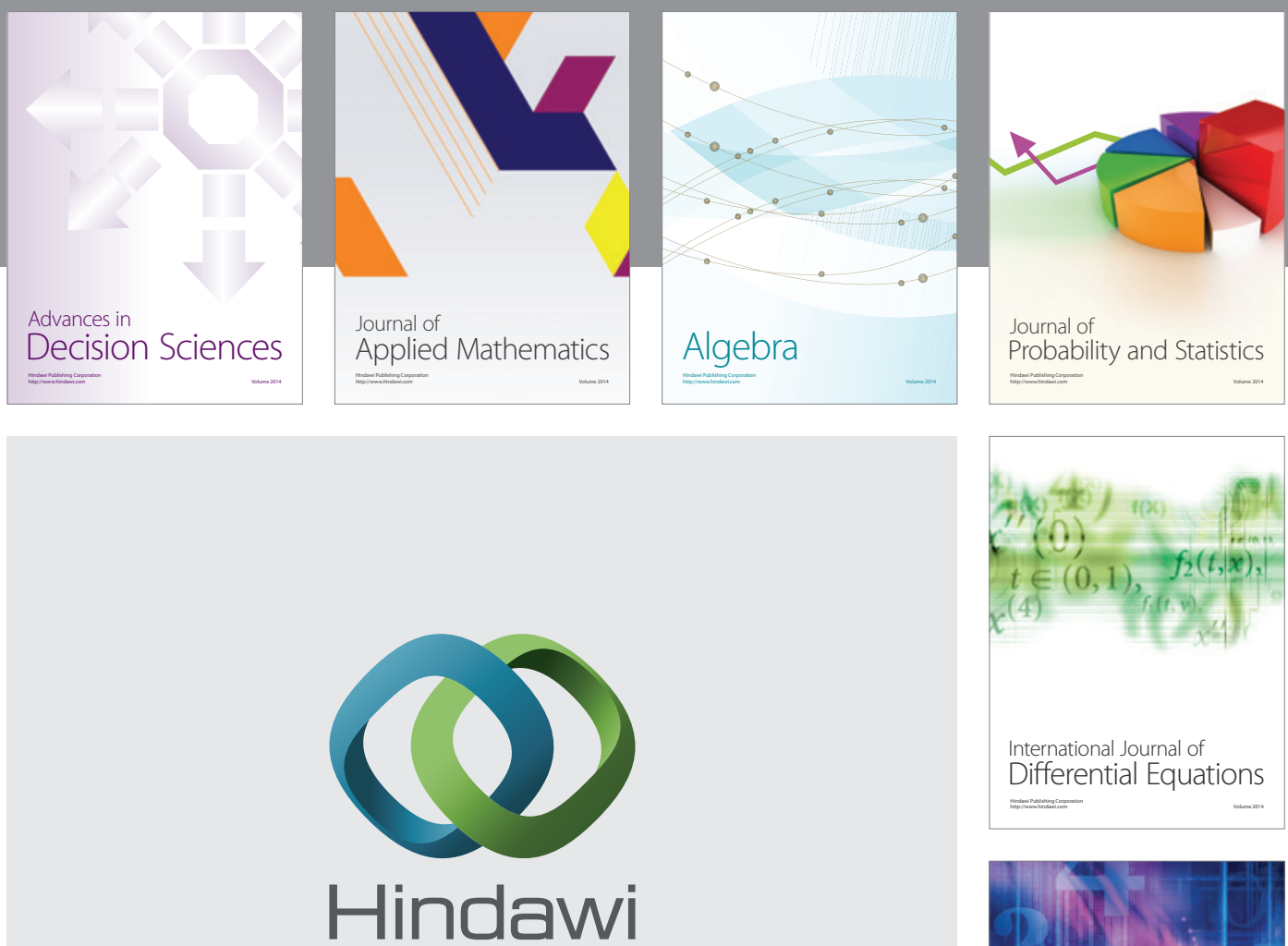

Submit your manuscripts at http://www.hindawi.com
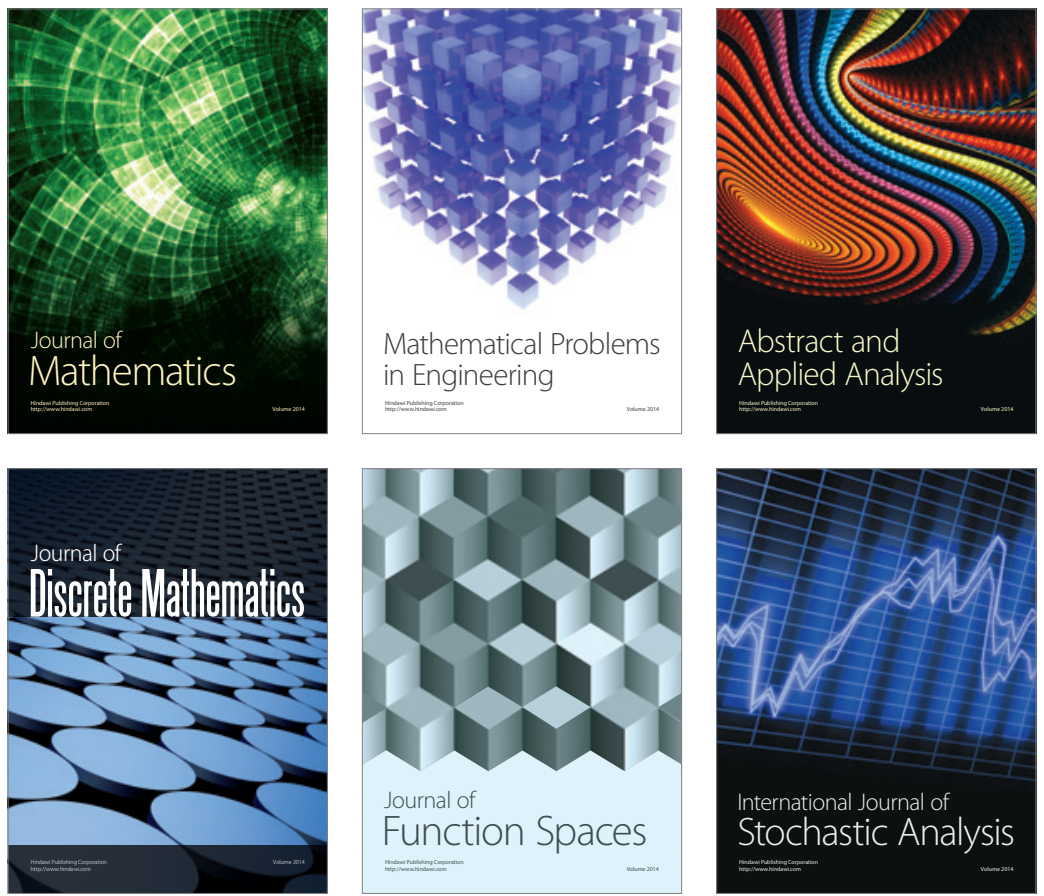

Journal of

Function Spaces

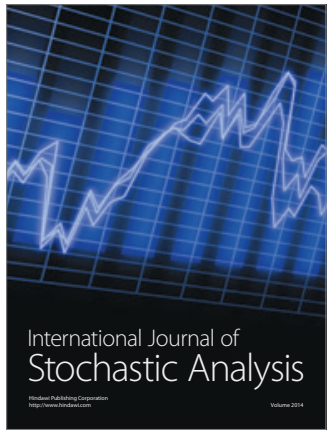

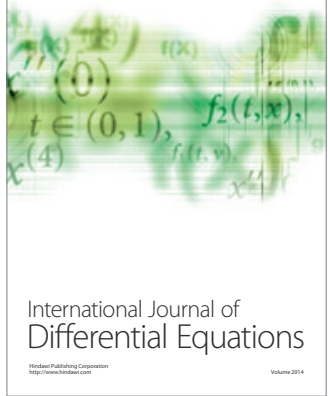
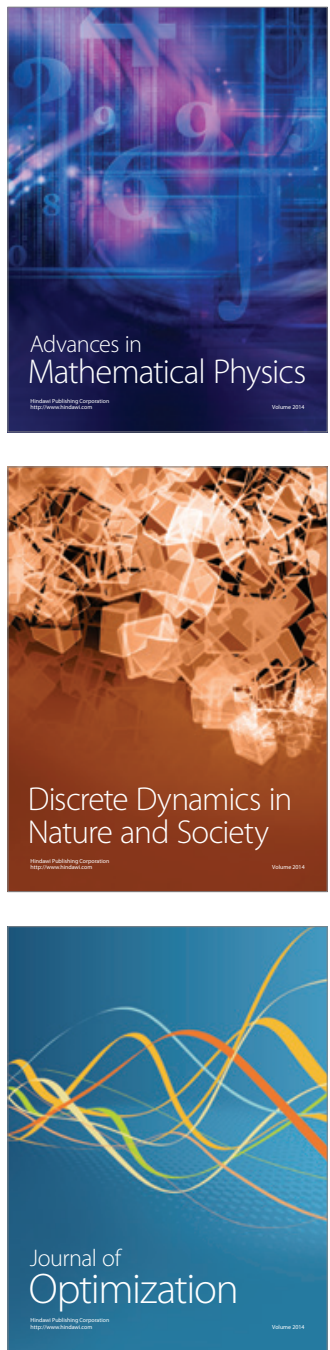